\title{
Impact of Shoulder Injuries on Quality of Life for Retired National Basketball Association Players: A Survey Study
}

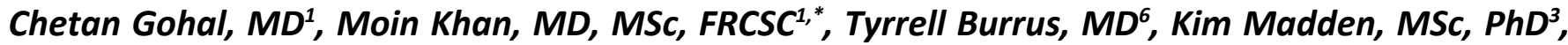 \\ Joel Gagnier, PhD2, Joseph P. Rogowski, MSc, ATC, CSCS ${ }^{4}$ and Asheesh Bedi, MD
}

\author{
${ }^{1}$ Division of Orthopaedic Surgery, McMaster University, Canada \\ ${ }^{2}$ Department of Orthopaedic Surgery, University of Michigan, Ann Arbor, Michigan, USA \\ ${ }^{3}$ Department of Health Research Methods, McMaster University, Canada \\ ${ }^{4}$ National Basketball Players Association, New York, United States
}

${ }^{5}$ MedSport, Department of Orthopaedic Surgery, University of Michigan, Ann Arbor, Michigan, USA

${ }^{6}$ Orthopedic Associates of Central Texas, TX, USA

*Corresponding author: Moin Khan, MD, MSC, FRCSC, Division of Orthopaedic Surgery, McMaster University, Hamilton, ON, Canada

\section{Abstract}

Background: Athletes in the National Basketball Association (NBA) are at risk of sport-related injuries due to the frequent jumping, pivoting, and overhead movements that constantly strain the musculoskeletal system. A comprehensive understanding of how shoulder injuries affect athletes during their NBA careers and into retirement has not been studied.

Purpose: The purpose of this study is to utilize a survey to evaluate the epidemiology of shoulder injuries in NBA players and determine the impact that these injuries have on post-retirement function and Health-Related Quality of Life (HRQOL).

Study design: Cross-sectional survey.

Methods: A survey encompassing demographic characteristics, injuries prior to and during a player's NBA career, and post-retirement Tegner Activity Levels and HRQOL as per the EQ-5D was developed and validated by a specialized focus group. It was distributed to all retired members of the National Basketball Players Association (NBPA) electronically through the SurveyMonkey platform, from which data was abstracted and analyzed. Descriptive statistics were used for means and proportions, dichotomous variables were compared with chi-squared test, continuous variables were compared with student's t-test, and non-parametric data was compared with Mann-Whitney $U$ test. A multiple regression analysis was performed to assess for potential factors correlated to injury.
\end{abstract}

Results: One hundred eight retired NBA players participated in the survey, with the majority being over the age of 55 (72.2\%, range $35-55+)$. A quarter of respondents $(27.6 \%)$ reported having a shoulder injury during their NBA career, most commonly shoulder dislocation $(37.9 \%)$ and acromioclavicular joint injury (37.9\%). Of those injured players, $72.4 \%$ continued to have shoulder pain until retirement. Over half of all respondents, $(51.9 \%)$ report current shoulder pain in the post-retirement period, which has significantly impaired their Tegner Activity Level $(p=0.003)$ and QOL as per the Visual Analog Scale $(p<0.00004)$.

Conclusion: A significant number of professional basketball players report shoulder injury during career, most commonly shoulder instability and AC joint injury. The majority of respondents continue to report pain until retirement. Post-retirement shoulder pain significantly impairs activity levels and HRQOL. Further evidence is needed to identify the risk factors for post-retirement shoulder pain to help guide clinicians in managing and counseling professional basketball players.

\section{Keywords}

NBA, Knee, Injury, Professional athlete

\section{Introduction}

The high intensity of professional level sports underlies their value as worldwide entertainment, but also pre-disposes the athletes to game-related injuries.

Citation: Gohal C, Khan M, Burrus T, Madden K, Gagnier J, et al. (2019) Impact of Shoulder Injuries on Quality of Life for Retired National Basketball Association Players: A Survey Study. Int J Sports Exerc Med 5:154. doi.org/10.23937/2469-5718/1510154

Accepted: December 02, 2019; Published: December 04, 2019

Copyright: (c) 2019 Gohal C, et al. This is an open-access article distributed under the terms of the Creative Commons Attribution License, which permits unrestricted use, distribution, and reproduction in any medium, provided the original author and source are credited. 
Athletes in the National Basketball Association (NBA) are at risk of sport-related injuries due to the frequent jumping, pivoting, and overhead movements that constantly strain the musculoskeletal system [1]. Although lower extremity injuries predominate in professional basketball, shoulders are the second most common upper extremity injury [1]. Shoulder injury in professional athletes can be devastating; for instance, a systematic review and meta-analysis by Klouche found that only $49.9 \%$ of competitive and professional athletes (from various sports) returned to equivalent level of play after rotator cuff repair [2]. Significant research has been performed on shoulder injuries in overhead athletes, with particular focus on throwing sports such as baseball, however such rigorous assessment has not been performed for basketball.

It is, however, thoroughly understood that various shoulder pathologies and associated pain may impact Quality of Life (QOL) and function. MacDermid, et al. identified rotator cuff pathology to be highly predictive of impaired physical health QOL [3]. Similarly, chronic shoulder impingement has been shown to result in significant functional disability and reduction in QOL [4]. The impact of shoulder pathology on an individual's perception of QOL can be comparable to the effects of hypertension, congestive heart failure, diabetes mellitus, and clinical depression [5]. Evidently, these are not injuries that should be ignored or overlooked.

Although in-game injuries in professional athletes are frequently witnessed and broadly displayed in the media, the sequelae of these injuries, especially post-retirement, have not been evaluated for NBA players. Insight into the impact of shoulder injuries on the quality of life and function of retired NBA players is lacking. Understanding the influence of these injuries on players' QOL and decision to retire can help managing physicians provide comprehensive treatment and mitigate risk factors. The purpose of this study is to survey retired NBA players to outline the epidemiology of shoulder injuries in these athletes and the treatments obtained, and to examine the impact of shoulder injuries on their daily function and quality of life post-retirement.

\section{Methods}

\section{Ethics review}

This study was approved by the University of Michigan Research Ethics Board.

\section{Survey development and validation}

A focus group was formed to collectively identify the appropriate components of the survey that would be used to evaluate musculoskeletal injury in the NBA and associated sequelae. The group consisted of orthopaedic surgeons that were fellowship-trained in sports medicine, team physicians, and a statistician. Athletic trainers from the NBA were also consulted regarding the appropriateness and comprehensiveness of the survey questions. The categories created include demographic characteristics, injuries prior to joining the NBA, injuries during the NBA career, and post-retirement treatments, function, and Health-Related Quality of Life (HRQOL). The primary locations of injury focused on knee, hip, and shoulder. Surgeons and athletic trainers were repeatedly surveyed until no new questions for the survey were generated.

To ensure face validity, the survey was pretested with two independent orthopaedic surgeons and a former professional basketball player to assess the appropriateness of the questions to adequately evaluate musculoskeletal health and injury in the NBA. Orthopaedic surgeons who specialize in treating professional basketball players assessed the survey for content validity and the questions were tailored based on their feedback. After adjustments to user-friendliness, comprehensiveness, and clarity were made, the final questionnaire consisted of sixty-one items. It included the Tegner Activity Level Scale [6], EQ-5D [7], and Visual Analog Scale (VAS) [7] to measure activity level, QOL, and overall health status, respectively.

\section{Survey administration}

The survey was distributed to all members of the National Basketball Players Association (NBPA) via electronic mail. It was accessible through the SurveyMonkey electronic survey platform and was available to be completed from April 1, 2017 to June 26, 2017. Respondents consisted only of NBA players who were retired at the time of survey distribution. Three reminder e-mails were sent by the NBPA to maximize response rate and electronic restrictions prevented more than one response from any one individual.

\section{Statistical analysis}

Data was extracted from the SurveyMonkey Platform into a Microsoft Excel (Version 14.0.0, Redmond, WA) spreadsheet and statistical analysis was performed using SPSS Statistics (Version 25, IBM, Armonk, NY). Summary statistics were presented as percentages and descriptive statistics were reported using median or mean. Dichotomous variables were presented as proportions and compared using a Chi-squared test. Continuous variables such as the VAS score were compared using the student's t-test. A Mann-Whitney $U$ test was used to compare all non-parametric data. For all statistical analysis a $p$-value of $<0.05$ was determined a priori as the threshold for statistical significance. Lastly, multiple regression analysis was performed to determine relationships between shoulder injury during the NBA career and post-retirement HRQOL, activity level, and overall health. 
Table 1: Demographic characteristics of respondents.

\begin{tabular}{|l|l|l|}
\hline Characteristics & Number (N = 108) & \% \\
\hline Age & & \\
$35-44$ & 10 & $9.3 \%$ \\
$45-54$ & 20 & $18.5 \%$ \\
$55+$ & 78 & $72.2 \%$ \\
\hline Years in NBA & & \\
$0-4$ & 27 & $25.0 \%$ \\
$5-9$ & 34 & $31.5 \%$ \\
$10-14$ & 39 & $36.1 \%$ \\
$15-19$ & 8 & $7.4 \%$ \\
\hline Primary Playing Position & & \\
Point Guard & 18 & $16.7 \%$ \\
Shooting Guard & 15 & $13.9 \%$ \\
Small Forward & 19 & $17.6 \%$ \\
Power Forward & 25 & $23.2 \%$ \\
Centre & 31 & $28.7 \%$ \\
\hline
\end{tabular}

\section{Results}

\section{Demographics}

A total of one hundred and eight retired players from the National Basketball Association responded to the survey, with $72.2 \%$ being over 55 years of age (range 35-55+). All five player positions were represented in the sample, with centres being the most common respondents $(N=31,28.7 \%)$ and shooting guards being the least common ( $N=15,13.9 \%)$. Sixty-eight percent of respondents had played between 5-14 years in the NBA (range 0-19 years) Table 1.

\section{Injuries prior to NBA career}

Approximately a third of respondents ( $N=35,32.4 \%)$ had a significant orthopaedic injury prior to beginning their NBA career. Of these players, only four $(12.5 \%)$ reported having a previous injury to their shoulder. Athletes with shoulder injuries prior to the NBA had trended towards having slightly higher rates of shoulder pain after retirement compared to those without prior shoulder injuries. (75\% vs. $\left.67.7 \%, X^{2}=0.09, p=0.77\right)$.

\section{Shoulder injuries during NBA career}

A total of 29 respondents (27.6\%) reported shoulder injury during NBA career, with some players experiencing more than one type of shoulder injury. The distribution of shoulder injuries included labrum tears $(N=5,17.2 \%)$, rotator cuff tears $(N=10,34.5 \%)$, proximal biceps tendon injury $(\mathrm{N}=3,10.3 \%)$, acromioclavicular joint injury ( $\mathrm{N}=11,37.9 \%)$, and shoulder dislocation ( $N=11,37.9 \%)$. Only four players $(13.8 \%)$ reported requiring surgery for their injury during their NBA career, which included three labrum repairs for instability (10.3\%) and one rotator cuff repair (3.4\%). For players who dislocated their shoulder, $27.2 \%(\mathrm{~N}=$ 3 ) underwent some form of shoulder surgery in comparison to $5.5 \%(\mathrm{~N}=1)$ of players who did not previ-
Table 2: Characteristics of shoulder injury during NBA career.

\begin{tabular}{|l|l|}
\hline & Number (\%) \\
\hline Any shoulder injury & $29 / 105(27.6)$ \\
\hline $\begin{array}{l}\text { Type of shoulder injury } \\
\text { Labrum Tear }\end{array}$ & $5(17.2 \%)$ \\
Rotator Cuff Tear & $10(34.5 \%)$ \\
Proximal biceps tendon injury & $3(10.3 \%)$ \\
\hline $\begin{array}{l}\text { Acriomioclavicular joint injury } \\
\text { Shoulder dislocation }\end{array}$ & $11(37.9 \%)$ \\
\hline Steroid injections & $11(37.9 \%)$ \\
\hline Surgery & $8(27.6)$ \\
\hline Any Surgery & \\
\hline Type of Surgery & $4(13.7)$ \\
\hline Labrum Repair & \\
Rotator Cuff Repair & $3(10.3)$ \\
\hline Outcomes & $1(3.4)$ \\
\hline $\begin{array}{l}\text { Games Missed } \\
\text { None } \\
<5\end{array}$ & $11(37.9)$ \\
$6-20$ & $9(31.0)$ \\
$21-50$ & $7(24.1)$ \\
$>$ & $1(3.5)$ \\
\hline Returned to play at same level & $1(3.5)$ \\
\hline Shoulder pain until retirement & $22(75.9)$ \\
\hline Retired because of shoulder & $21(72.4)$ \\
\hline $\begin{array}{l}\text { Would have played longer if no shoulder } \\
\text { pain }\end{array}$ & $2(6.9)$ \\
\hline & $13(44.8)$ \\
\hline
\end{tabular}

${ }^{*}$ Respondents can select more than one response; $N=29$ unless otherwise specified.

ously dislocate their shoulder $\left(X^{2}=2.7, p=0.10\right)$. Of the players with shoulder injuries, eight $(27.6 \%)$ were treated at some point with a corticosteroid injection into their shoulder. Players were significantly more likely to require a shoulder injection if they injured their shoulder during their career $\left(24.1 \%\right.$ vs. $8.0 \%, X^{2}$ $=4.9, p=0.026$ ).

Almost one-fifth of players missed career games due to shoulder injury (17\%), with one player missing an entire season. A quarter of players with shoulder injuries did not return to the same level of play $(\mathrm{N}=$ $7,24.1 \%)$. Three quarters of players who had a shoulder injury during their career stated their shoulder continued to bother them until retirement $(\mathrm{N}=21$, $72.4 \%)$, and almost half of them stated they would have continued playing if they did not have shoulder pain ( $N=13,44.8 \%)$. Despite this, only two players $(6.9 \%)$ actually reported that they retired due to their shoulder injury Table 2.

\section{Post-retirement shoulder health}

Half of the respondents reported currently having shoulder pain ( $\mathrm{N}=54,51.9 \%$ ), and thirteen (12.5\%) required shoulder corticosteroid injections following retirement from the NBA. Five players $(4.8 \%)$ have re- 
Table 3: Detailed responses to HRQOL measures.

\section{EQ-5D Domains and VAS}

\begin{tabular}{|l|l|}
\hline & $\begin{array}{l}\text { Number (\%) } \\
\text { N = 108 }\end{array}$ \\
\hline Mobility & \\
No problems & $45(41.7)$ \\
Slight problems & $23(21.3)$ \\
Moderate problems & $25(23.1)$ \\
Severe problems & $6(5.6)$ \\
Unable to walk & $1(0.9)$ \\
Missing & $8(7.4)$ \\
\hline
\end{tabular}

\section{Self Care}

No problems

$88(81.5)$

Slight problems

Moderate problems

Severe problems

$9(0)$

Unable to wash or dress myself

Missing

$8(7.4)$

Usual Activities

No problems

$48(44.4)$

Slight problems

$32(29.6)$

$15(13.9)$

$0(0)$

Severe problems

$4(3.7)$

Unable to do my usual activities

9 (8.3)

Missing

$12(11.1)$

No pain

$40(37.0)$

Slight pain

Moderate pain

4 (3.7)

Severe pain

$2(1.9)$

Extreme pain

$8(7.4)$

Anxiety/Depression

No anxiety or depression

56 (51.9)

Slight anxiety or depression

Moderate anxiety or depression

$14(13.0)$

Severe anxiety or depression

Extreme anxiety or depression

$0(0)$

Missing

$8(7.4)$

VAS Current Health (0 to 100)

Mean 74.1 (SD 17.1)

Tegner Activity Level Scale

Current activity level

\begin{tabular}{l|l} 
Level 10 & $0(0)$ \\
Level 9 & $2(1.9)$ \\
Level 8 & $1(0.9)$ \\
Level 7 & $5(4.6)$ \\
Level 6 & $16(14.8)$ \\
Level 5 & $9(8.3)$ \\
Level 4 & $10(9.3)$ \\
Level 3 & $16(14.8)$ \\
Level 2 & $22(20.4)$ \\
Level 1 & $6(5.6)$ \\
Level 0 & $4(3.7)$ \\
Missing & $17(15.7)$
\end{tabular}

quired shoulder surgery since retirement, of which two players required multiple surgeries. Interestingly, none of these five players indicated that they had a shoulder injury during their NBA career. The surgeries included two shoulder arthroscopies and loose body removal, two rotator cuff repairs, and one labral repair. Players were significantly more likely to have shoulder pain post-retirement if they injured their shoulder during their career $\left(69.0 \%\right.$ vs. $\left.45.3 \%, X^{2}=4.7, p=0.031\right)$.

\section{Post retirement quality of life}

The overall median Tegner Activity Level for all respondents was Level 3, which corresponds to "work light labour". Almost one-third of respondents reported moderate $(N=25,25 \%)$ to severe $(N=6,6 \%)$ impairments in mobility on the EQ-5D. Self-care was typically not on issue for respondents, with $88 \%$ of respondents having no difficulty and only 1 player (1\%) unable to provide self-care. Over half of respondents had some level of difficulty with usual activities ( $N=51,51.5 \%$ ). Most retired NBA players in the survey had some pain or discomfort ( $\mathrm{N}=88,88.0 \%)$, with almost half $(\mathrm{N}=48$, $48.0 \%)$ rating it between moderate and extreme. Similarly, almost half of all respondents reported experiencing some level of anxiety or depression ( $N=44,44.0 \%)$. The average rating for current health by respondents was 74.1 17.1 on the EQ-5D VAS scale Table 3.

There was no significant difference identified in median Tegner Activity Level between athletes who reported shoulder injury during their NBA career and those who did not (median $=4$ vs. $3, Z=0.47, p=$ 0.31). However, athletes who had current shoulder pain post-retirement had significantly lower Tegner Activity Levels than those without shoulder pain (median $=2.5$ vs. $4, Z=2.72, p=0.003$ ). Respondents with current shoulder pain also had significantly worse mobility (median $=3$ vs. $5, \mathrm{Z}=4.25, \mathrm{p}=<0.00001$ ), ability to perform usual activities (median $=4$ vs. $5, Z$ $=3.75, p=0.00009$ ), and anxiety/depression (median $=4$ vs. $5, Z=2.88, p=0.002$ ). The players with post-retirement shoulder pain rated their overall health at a mean of $68.0 \pm 19.3$, which was significantly lower than those without shoulder pain (mean $=81.4 \pm$ $10.4, p=0.00004)$. When adjusted for age and ethnicity, having a shoulder injury during one's NBA career did not have a significant impact on current activity level $(p=0.803)$ or current health $(p=0.131)$ Table 4 .

\section{Discussion}

Shoulder instability and acromioclavicular joint injuries are common in professional basketball athletes and affected over one-third of the survey participants in the current study. Furthermore, the players were significantly more likely to have shoulder pain post-retirement if an injury was sustained during professional career. Further, NBA players who have current shoulder pain post-retirement reported signifi- 
Table 4: Results of Mann-Whitney U test comparing EQ-5D subscales between athletes with post-retirement shoulder pain to those without.

\begin{tabular}{|l|l|l|l|l|}
\hline EQ-5D Subscale & Median & \multicolumn{2}{|l|}{} & Z \\
\hline & Post-retirement shoulder pain & No post-retirement shoulder pain & & \\
\hline Mobility & 3 & 5 & 2.9 & $0.002^{*}$ \\
\hline Self-Care & 5 & 5 & 1.9 & $0.02^{*}$ \\
\hline Usual Activities & 4 & 5 & 3.8 & $0.00009^{*}$ \\
\hline Pain/Discomfort & 3 & 4 & 4.2 & $<0.00001^{*}$ \\
\hline Anxiety/Depression & 4 & 5 & 2.9 & $0.002^{*}$ \\
\hline
\end{tabular}

*Statistically significant difference.

cantly lower Tegner activity levels, and also reported significant impairments in all HRQOL components of the EQ-5D: Mobility, pain/discomfort, anxiety/depression, and ability to provide self-care and perform usual activities. The number of players impacted by this is far from negligible, as over half of respondents reported currently having shoulder pain.

Interestingly, the proportion of players with shoulder pain post-retirement was almost double the proportion of players reporting shoulder injury in the NBA (51.9\% vs. $27.6 \%)$. This may be explained in part by degenerative changes of the shoulder that occur with aging and contribute to shoulder pain in the elderly, as opposed to sequelae of acute sport-related injuries. A review and cross-sectional survey by Burner, et al. found that on average $31 \%$ of elderly patients (age $>60$ ) report severe shoulder pain [8] and a systematic review by Luime, et al. reports the prevalence of shoulder pain in the adult population to range from $6.9-31 \%$ [9]. Despite the majority of respondents to the study survey being over the age of 55 (72.2\%), which could attribute some aspects of their shoulder pain to degenerative changes, the overall prevalence of current shoulder pain in this group was even higher than in the elderly population (51.9\% vs. $31 \%)$. With prevalence this high, it becomes essential to delineate risk factors, define etiologies, and devise new management strategies to mitigate post-retirement shoulder pain and subsequent impairment in $\mathrm{HRQOL}$ for NBA players.

Not only were players with shoulder injuries during career more likely to have shoulder pain post-retirement, the injuries also impacted playing during professional play. Almost one-fifth of players missed career games due to shoulder injury and a quarter (24.1\%) of players felt that they did not return to the same level of play afterwards. Optimal performance is pivotal to the success of a professional athlete's career, highlighting the importance of minimizing injury and maximizing healthy playing time. This is proving difficult for players with shoulder injuries, as three quarters $(72.4 \%)$ reported shoulder complaints until retirement. A player's perception of injury plays an important role in potentially negative influence on performance due to concepts such as re-injury anx- iety [4], where a player cannot perform at an optimal level due to fear of repeat injury. Furthermore, chronic injuries in elite athletes have been demonstrated to result in significant psychological impacts on elite athletes, contributing to anxiety, depression, and low self-esteem [10]. The current study supports this in that players with NBA shoulder pain or injury had significantly higher levels of anxiety/depression than players without injury. Further research can be done to evaluate the influence of these chronic shoulder issues on player performance based on in-game statistics, as well as develop strategies to reduce the chronic nature of these injuries.

In the current study, four players required surgical management for shoulder injuries during professional play, implying the remaining injured players who sought treatment were managed non-operatively. However, as mentioned, a significant percentage continued to report shoulder pain until retirement. This raises the question as to whether players did not seek out medical treatment due to belief that injury was insignificant, or whether non-operative rehabilitation protocols were inadequate. Therefore, non-operative rehabilitation protocols can be evaluated to determine which components are effective and which are not, with the aim to reduce the number of athletes playing with chronic shoulder pain. Counseling and treatment for players should focus on long-term shoulder health and overall function, as players are likely to experience shoulder pain post-retirement than during their NBA career.

The strengths of this paper include the rigorous methodology used to develop the survey. A diverse focus group with specialized Orthopaedic surgeons and sports physicians ensured a comprehensive questionnaire was produced to encapsulate relevant musculoskeletal injuries in the NBA. The study provides information and insight on patient-important outcomes in the retired NBA population, which is a group whose injuries and consequent $\mathrm{QOL}$ has not been thoroughly evaluated.

A limitation of the study is that the survey focuses on a very specific, all-male, professional level athlete population for one sport, limiting the generalizability of 
the results. Having a very specific population also limited the sample size for the study. The survey also does not provide a temporal relationship between injuries and treatments obtained. For instance, there is data on whether a player has dislocated their shoulder or received a corticosteroid injection, but the timing and number of dislocations or injections is unknown. These factors can influence management strategies by treating physicians.

Future studies can identify the major risk factors for NBA players that contribute to post-retirement shoulder pain so that preventative measures can be developed to reduce the burden on HRQOL. Further high-quality studies, such as long-term prospective cohort studies, are also needed to more thoroughly evaluate musculoskeletal health and HRQOL of retired professional athletes.

\section{Conclusion}

A significant number of professional basketball players report shoulder injury during career, with the majority of respondents reporting pain until retirement. Post-retirement shoulder pain significantly impairs activity levels and HRQOL. Further evidence is needed to identify the risk factors for post-retirement shoulder pain to help guide clinicians in managing and counseling professional basketball players.

\section{References}

1. Drakos MC, Domb B, Starkey C, Callahan L, Allen AA (2010) Injury in the National Basketball Association: A 17year overview. Sports Health 2: 284-290.

2. Klouche S, Lefevre N, Herman S, Gerometta A, Bohu Y (2016) Return to Sport After Rotator Cuff Tear Repair: A
Systematic Review and Meta-analysis. Am J Sports Med 44: 1877-1887.

3. MacDermid JC, Ramos J, Drosdowech D, Faber K, Patterson S (2004) The impact of rotator cuff pathology on isometric and isokinetic strength, function, and quality of life. $J$ Shoulder Elbow Surg 13: 593-598.

4. Chipchase LS, O'Connor DA, Costi JJ, Krishnan J (2000) Shoulder impingement syndrome : Preoperative health status. J Shoulder Elbow Surg 9: 12-15.

5. Ware JE, Kosinski M, Keller SD (1993) SF-36 physical and mental health summary scales: a user's manual. In: The Health Institute, New England Medical Center, Boston 1994: 1.1-10.12.

6. Collins NJ, Misra D, Felson DT, Crossley KM, Roos EM (2011) Measures of knee function: International Knee Documentation Committee (IKDC) Subjective Knee Evaluation Form, Knee Injury and Osteoarthritis Outcome Score (KOOS), Knee Injury and Osteoarthritis Outcome Score Physical Function Short Form (KOOS-PS), Knee Outcome Survey Activities of Daily Living Scale (KOS-ADL), Lysholm Knee Scoring Scale, Oxford Knee Score (OKS), Western Ontario and McMaster. Arthritis Care Res 63: 208-228.

7. Janssen B, Szende A (2013) Population Norms for the EQ5D. Self-Reported Population Health: An International Perspective Based on EQ-5D.

8. Burner T, Abbott D, Huber K, Stout M, Fleming R, et al. (2014) Shoulder symptoms and function in geriatric patients. J Geriatr Phys Ther 37: 154-158.

9. Luime J, Koes B, Hendriksen IJ, Burdorf A, Verhagen AP, et al. (2004) Prevalence and incidence of shoulder pain in the general population; a systematic review. Scand J Rheumatol 33: 73-81.

10. Von Rosen $P$, Kottorp A, Fridén C, Frohm A, Heijne A (2018) Young, talented and injured: Injury perceptions, experiences and consequences in adolescent elite athletes. Eur J Sport Sci 18: 1-10. 